\title{
Local mechanical properties of bladder cancer cells measured by $A F M$ as a signature of metastatic potential ${ }^{\star}$
}

\author{
Y. Abidine ${ }^{1,2, a}$, V.M. Laurent ${ }^{1,2}$, R. Michel ${ }^{1,2}$, A. Duperray ${ }^{3,4}$, and C. Verdier $^{1,2, b}$ \\ 1 Univ. Grenoble Alpes, LIPHY, F-38000 Grenoble, France \\ 2 CNRS, LIPHY, F-38000 Grenoble, France \\ 3 INSERM, IAB, F-38000 Grenoble, France \\ 4 Univ. Grenoble Alpes, IAB, F-38000 Grenoble, France
}

Received: 15 June 2015 / Revised: 15 August 2015

Published online: 13 October 2015

(C) The Author(s) 2015. This article is published with open access at Springerlink.com

\begin{abstract}
The rheological properties of bladder cancer cells of different invasivities have been investigated using a microrheological technique well adapted in the range $[1-300 \mathrm{~Hz}]$ of interest to understand local changes in the cytoskeleton microstructure, in particular actin fibres. Drugs disrupting actin and actomyosin functions were used to study the resistance of such cancer cells. Results on a variety of cell lines were fitted with a model revealing the importance of two parameters, the elastic shear plateau modulus $G_{N}^{0}$ as well as the glassy transition frequency $f_{\mathrm{T}}$. These parameters are good markers for invasiveness, with the notable exception of the cell periphery, which is stiffer for less invasive cells, and could be of importance in cancer metastasis.
\end{abstract}

\section{Introduction}

Cancer cell metastasis is a multi-stage process characterized by cell malfunctional behavior. Some of the major pathological characteristics of cancer cells are their particular mechanical properties, and their ability to invade surrounding tissues, transmigrate and proliferate at new sites. A critical step in cancer is the conversion from non-tumorigenic cell to metastatic cell, and it is not yet understood how this change occurs. However, there are evidences that cancer progression is characterized by disruption and reorganization of the actin cytoskeleton [1] as well as changes in the mechanical properties [2]. This change is probably associated with the enhanced capability of cancer cells to migrate and adapt to changing environments.

The mechanical properties of cells are essentially determined by the cytoskeleton microstructure [3-5] which is remodeled during cell migration, adhesion, proliferation and differentiation. They are essential for the regulation of cell functions. Several studies of a variety of diseases using different experimental techniques have shown that abnormalities are connected with the mechanical properties of cells, in particular in cancer metastasis [6,7]. In fact, this is a subject of controversy as some studies report a stiffening of cancer cells compared to benign cells [8], while others show that cancer is characterized by the decrease of the cell stiffness $[9,10]$ i.e. metastatic cancer cells have an elastic modulus lower than that of healthy cells. It has also been suggested that variations of dynamical properties taking place during tumour invasion provide a signature of malignancy [11].

The determination of mechanical properties of single cells has become possible with the development of local measurement techniques such as magnetic twisting cytometry [12], laser tracking microrheology [13], two-point microrheology [14] or the atomic force microscope (AFM) $[15,16]$. Some researchers [9] investigated the elastic response of normal and human bladder cancer cells using the AFM. They found that cancer cells exhibit a Young modulus lower than normal cells. This softening of cancer cells has also been observed with other techniques such as the optical stretcher [17]. AFM has proved to be a reliable tool to probe the load-displacement relationship of soft materials at small scale with high resolution. It is especially valuable to characterize static and frequency-dependent mechanical

\footnotetext{
* Contribution to the Focus Point on "The Physics of Cancer" edited by M. Ben Amar.

a e-mail: yara.abidine@gmail.com

b e-mail: claude.verdier@ujf-grenoble.fr
} 
properties of biological specimens. Thus, AFM is becoming one of the most popular tools to measure cell mechanical properties [18-20].

The cytoskeleton, comprised of actin filaments, intermediate filaments and microtubules is a key element for regulating these mechanical properties. In particular, the active forces generated by the actin cytoskeleton can modify or even control them [21]. Their effect on self-organization and material properties has been studied extensively [22, 23] as mechanical measurements can be obtained together with the analysis of intracellular movement [24]. Disrupting the actin network results in a softening of cells [25-27]. Indeed, the acto-myosin contractility is essential for the production of cellular tensions. Inhibiting these major functions is often used to characterize the relationship between actin microstructure and mechanical properties. In particular, actin polymerization drives cancer cell motility [28]. A way to inhibit the actin polymerization is to use Latrunculin A, a powerful drug that binds to the G-actin monomers and prevents them from polymerizing with the actin fibres [29]. The ability of cancer cells to migrate requires force generation to overcome factors that oppose movement. F-actin assembles with myosin II filaments to form a protein complex that uses energy from the ATP hydrolysis to power actin-myosin contraction [30]. Y27632 is a drug inhibiting the Rho-kinase complex which is essential for contractility. It will also induce a relaxation of the cytoskeleton [31].

In this study, we report on the viscoelastic properties of human bladder cancer cells by dynamic indentation experiments using AFM. We investigate the complex shear modulus of three different cell lines with different metastatic potential. We probe the elastic and viscous moduli at three different locations across the cell: nucleus, perinucleus and the cell periphery. With the use of actin inhibitory drugs, we correlate mechanical properties and the actin microstructure obtained by confocal microscopy imaging. We propose a simplified multi power-law model to describe the behavior of the elastic and viscous moduli. We also report a relationship between the malignancy of cancer cells and their viscoelastic properties. In particular, we find that the elastic plateau modulus and the transition frequency (frequency at which $G^{\prime}=G^{\prime \prime}$ ) can be used as markers of invasiveness.

\section{Measurements of the local viscoelasticity using AFM}

\subsection{Experimental setup}

Experiments were carried out using a Nanowizard II AFM (JPK Instruments, Berlin, Germany) mounted on a Zeiss microscope (Observer D1, Carl Zeiss, Jena, Germany). All measurements were carried out in Force Modulation Mode at $37^{\circ} \mathrm{C}$ using the Petri Dish Heater (JPK Instruments, Berlin, Germany). We used a triangular silicon nitride cantilever with a four-sided pyramidal tip (MLCT, Bruker, lever C) with a nominal spring constant $k=0.01 \mathrm{~N} \mathrm{~m}^{-1} \mathrm{calibrated}$ using the thermal noise method. Sharp tips are better suited to reach a higher spatial resolution. We keep the applied force lower than $3 \mathrm{nN}$ to remain in the linear elastic regime where the Hertz model can be used. When the cantilever tip gets into contact with the sample, the force acting on the cantilever increases to a previously chosen setpoint $\left(F_{0}\right)$, corresponding to an initial indentation $\delta_{0}$. The relationship is given by Sneddon's modification of the Hertzian contact mechanics model [32]:

$$
F_{0}=\frac{3 E \tan \theta}{4\left(1-\nu^{2}\right)} \delta_{0}^{2}
$$

where $E$ is the cell's Young modulus, $\nu$ its Poisson ratio $(\nu \sim 0.5)$ and $\theta=20^{\circ}$ is the half pyramid angle. $\delta_{0}$ is chosen so that the tip penetration depth into the sample is large enough to have a sufficient contact area and not too large to remain within the linear elasticity regime. In order to carry out microrheology measurements, a small perturbation $\delta(\omega)$ (frequency $f$ from $1 \mathrm{~Hz}$ to $300 \mathrm{~Hz}$, and $\omega=2 \pi f$ is the angular frequency) was superposed to the initial indentation. During this procedure the tip remained in contact with the cell. We impose the indentation $\delta(\omega)$ and we measure the force response $F(\omega)$, as previously described [33]. The perturbation being small, eq. (1) can be linearized around the equilibrium. By the correspondence principle of linear viscoelasticity (LVE), in the $\omega$-domain, one operates with complex quantities, the indentation $\delta^{*}$ and the force $F^{*}$, and the hydrodynamic drag $i \omega b(0)$ is substracted [16]. The drag coefficient $b(0)$ is estimated from the extrapolation of the fit $b(h)$ as a function of the tip-sample separation $h$ to $h=0$. Then using the relationship $G^{*}=E^{*} /(2(1+\nu))$, the complex shear modulus $G^{*}(\omega)$ becomes:

$$
G^{*}(\omega)=\frac{1-\nu}{3 \delta_{0} \tan \theta}\left\{\frac{F^{*}(\omega)}{\delta^{*}(\omega)}-i \omega b(0)\right\} .
$$

From this relationship one can extract the real part (elastic modulus $G^{\prime}$ ) and imaginary one (loss modulus $G^{\prime \prime}$ ).

Cells were cultured on a glass substrate so there may be a possible influence of the substrate on thin cell regions. This is known as the "bottom effect" artefact that makes cell appear stiffer than they really are when thin samples are used. To avoid this artefact, usually the indentation is limited to $10 \%$ of the cell thickness [10]. In our study, we want to compare the local viscoelasticity of human bladder cancer cells, especially at the periphery, where the cell thickness is around $200 \mathrm{~nm}$. Thus, it is necessary to correct for substrate effects in AFM indentation measurements. 


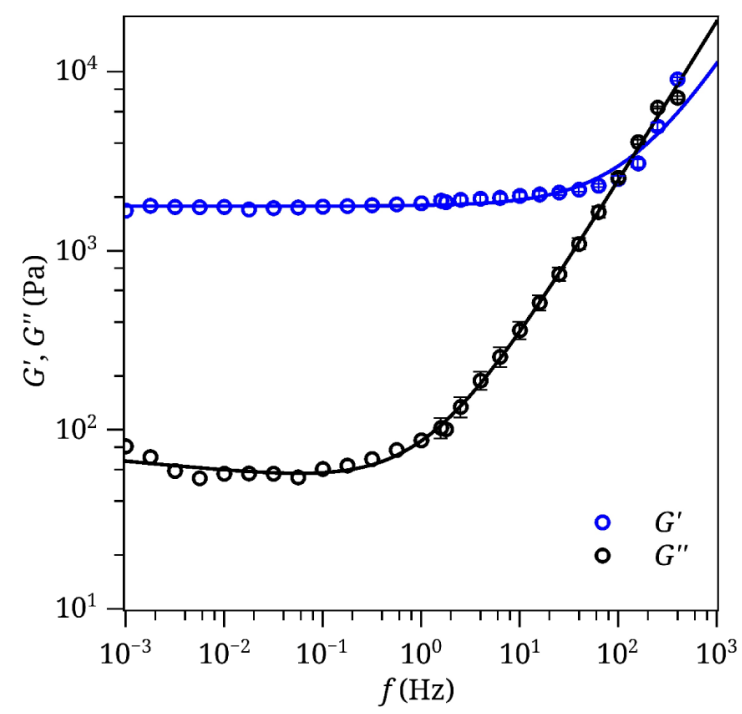

Fig. 1. Rheology of the $10 \%$ acrylamide gel: rheometrical and AFM experimental data are shown. Solid lines are the best model fit. Error bars represent SEM.

The effect of the substrate on thin films using spherical tips has been investigated previously [34,18]. Recently, new results were given [35] to investigate indentation of thin soft films using sharp conical indenters using finite element modeling. They propose a correction function $g_{B}\left(\chi_{0}\right)$ with $\chi_{0}=\left(\delta_{0} \tan \theta\right) / h$ including finite thickness effects and the load-displacement $\left(F-\delta_{0}\right)$ curves for conical indenters can be described by $F=F_{\text {Hertz }}\left(\delta_{0}\right) g_{B}\left(\chi_{0}\right)$, where $F_{\text {Hertz }}\left(\delta_{0}\right)$ is given by the usual Hertz model for conical tips, similar to eq. (1) expect for the leading coefficient $(2 / \pi$ instead of $3 / 4)$. This correction function was readily generalized into the frequency domain by linearizing with respect to $\delta(\omega)$, and the resulting complex shear modulus $G_{\mathrm{c}}^{*}$ probed by a pyramidal tip is given by

$$
G_{\mathrm{c}}^{*}(\omega)=\frac{1-\nu}{3 \tan \theta \delta_{0}} \frac{F^{*}(\omega)}{\delta^{*}(\omega)} g\left(\chi_{0}\right)^{-1}
$$

The correction function is estimated by

$$
g\left(\chi_{0}\right)=1+0.945 \chi_{0}+1.447 \chi_{0}^{2}+3.108 \chi_{0}^{3}-1.0668 \chi_{0}^{4}
$$

With this correction of the substrate effect on thin layers, we can probe the complex shear modulus locally in all cell regions.

\subsection{Rheological model}

This method was tested on polyacrylamide gels in previous work [33]. In the latter paper, a fractional derivative model was used to model the viscoelastic behavior of the gels in a wide frequency range. The model can be simplified here to account for the reduced frequency domain and the possible different slopes [36,37], thus the gel or the cell elastic and viscous moduli can be modelled as

$$
\begin{aligned}
G^{\prime}(\omega) & =G_{N}^{0}+k_{1} \omega^{a} \\
G^{\prime \prime}(\omega) & =k_{0} \omega^{-n_{\mathrm{f}}}+b k_{1} \omega^{a},
\end{aligned}
$$

where $G_{N}^{0}$ is the classical elastic plateau modulus at low frequencies, $-n_{\mathrm{f}}$ the slope of $G^{\prime \prime}$ at low frequencies in log-log plot, $a$ the exponent of the slope of $G^{\prime}$ and $G^{\prime \prime}$ in the glass transition regime, $b$ a parameter describing the ratio $G^{\prime \prime} / G^{\prime}$ at high frequencies, whereas $k_{0}$ and $k_{1}$ are two other fitting parameters.

Figure 1 shows the $G^{\prime}$ and $G^{\prime \prime}$ moduli of a polyacrylamide gel (10\% concentration) measured with a classical rheometer ( $f$ from $10^{-3} \mathrm{~Hz}$ to $3 \mathrm{~Hz}$ ) and by AFM in force modulation mode ( $f$ from $1 \mathrm{~Hz}$ to $300 \mathrm{~Hz}$ ). Fitting was achieved using the model eqs. (5)-(6) with parameters summarized in table 1 below.

A typical transition frequency $f_{\mathrm{T}}$ corresponding to the crossing of $G^{\prime}$ and $G^{\prime \prime}\left(G^{\prime}=G^{\prime \prime}\right)$ can also be deduced. Above this frequency, $G^{\prime}$ and $G^{\prime \prime}$ increase with a similar slope $a$. In our previous paper [33], we identified this frequency 
Table 1. Best-fitting values of parameters used in the model for the polyacrylamide gel.

\begin{tabular}{ccccccc}
\hline$G_{N}^{0}(\mathrm{~Pa})$ & $n_{\mathrm{f}}$ & $k_{0}(\mathrm{~Pa} \cdot \mathrm{s})$ & $k_{1}(\mathrm{~Pa} \cdot \mathrm{s})$ & $a$ & $b$ & $f_{\mathrm{T}}(\mathrm{Hz})$ \\
\hline 1773 & 0.05 & 51 & 3.8 & 0.89 & 2.0 & 158 \\
\hline
\end{tabular}

as a signature of the polyacrylamide gel. In the domain of high frequencies with $b>1$, the transition frequency $f_{\mathrm{T}}$ can be approximated by

$$
f_{\mathrm{T}}=\frac{1}{2 \pi}\left(\frac{G_{N}^{0}}{(b-1) k_{1}}\right)^{1 / a}
$$

The model (5)-(6) is quite successful for describing gel rheology, therefore it will serve as a basis for modelling of cell rheology, as discussed in the next part.

\section{Materials and methods}

\section{Bladder cancer cells and culture}

Three bladder cancer cell lines, representing different malignancy states of metastasis progression, were used, RT112, T24 and J82 (ATCC, Rockville, MD). RT112 cancer cells are moderately differentiated while T24 and J82 cancer cells are poorly differentiated and have a higher malignancy potential.

Cells were cultured in RPMI 1640 supplemented with 10\% fetal calf serum and 1\% penicillin streptomycin. One day before measurements, cells were seeded at a density of $3 \times 10^{4}$ cells per well on glass coverslips coated with $25 \mu \mathrm{g} / \mathrm{mL}$ fibronectin overnight at $37^{\circ} \mathrm{C}$ in humidified $5 \% \mathrm{CO}_{2}$ atmosphere. Such cells are considered to be in a low migrating state since the fibronectin concentration is quite high [38]. AFM measurements were carried out on isolated cells at $37^{\circ} \mathrm{C}$. Three independent preparations were made for each cell line. Cancer cell lines were transfected with the LifeAct plasmid expressing actin-GFP (Green Fluorescent Protein - pEGFP).

\section{Inhibition of the actin functions}

In order to verify how the actin cytoskeleton influences the viscoelastic properties, T24 cells were treated with two inhibitory drugs. For inhibition of the F-actin polymerization, cells were treated with $1 \mu \mathrm{M}$ Latrunculin A (SigmaAldrich) and a Rho-associated protein kinase (ROCK) inhibitor Y27632 (20 $\mu \mathrm{M}$, Sigma-Aldrich) was used to inhibit the actomyosin contractility. Measurements were done before and after treatment. Once the first measurements of $G^{\prime}$ and $G^{\prime \prime}$ were made, the medium was removed and a new medium containing the drug was added at the desired concentration. Measurements were then carried out every $10 \mathrm{~min}$. In this study we report only the measurements at $t=0$ and $t=20 \mathrm{~min}$. It is shown that the effect of the treatment is stable over $30 \mathrm{~min}$ and reversibility was verified.

\section{Confocal measurements}

Confocal microscopy was used separately to determine the microstructure of the actin cytoskeleton before and after inhibition of the actin polymerization and acto-myosin contractility. Confocal image stacks (Z-stacks) were acquired with a Leica TCS SP8 using Argon laser (488 nm) and a 40x immersion objective.

To quantify the distribution of the actin fibres in the cell, the mean fluorescence intensity of three regions of the cell (nucleus, perinucleus and periphery) was analyzed using the Image J software ${ }^{1}$. Regions of interest were defined by dividing the entire z-stack of confocal images into three sub-stacks where the mean fluorescence intensity is then calculated.

\section{Stastistical analysis}

Data were analyzed by a two-sample unpaired Student's t-test. Values correspond to $p<0.001(* * *), p<0.01(* *)$, $p<0.05\left(^{*}\right)$ and $p>0.05$ (n.s.). Values were considered statistically significant when $p<0.01$. 


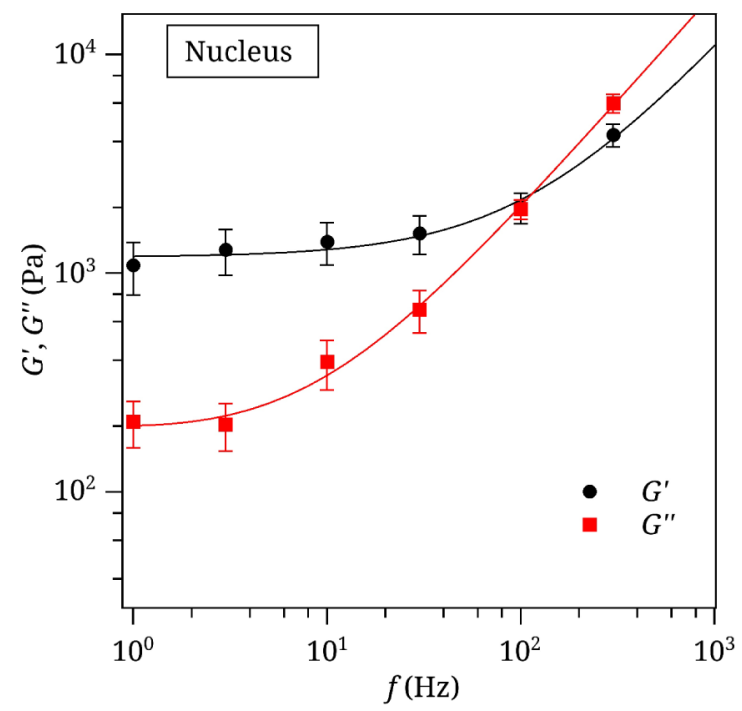

Fig. 2. Evolution of moduli $G^{\prime}$ and $G^{\prime \prime}$ in a region above the nucleus of individual T24 cells $(N=20$, error bars represent SEM). Curves were fitted with eqs. (5)-(6) and parameters are discussed below.

\section{Results and discussion}

\subsection{Bladder cancer cell T24 and spatial dependency}

Viscoelastic properties were probed on T24 cancer cells locally, at three different locations across the cell: nucleus (N), perinucleus $(\mathrm{P})$ and the edge of the cell $(\mathrm{E})$. For viability reasons, measurements were carried out at 6 frequencies only: $f=1,3,10,30,100,300 \mathrm{~Hz}$. Let us first concentrate on one location, as shown in fig. 2 representing the elastic $G^{\prime}$ and viscous moduli $G^{\prime \prime}$ as a function of frequency, measured above the nucleus.

The complex shear modulus follows the typical frequency dependence found for other cells such as neutrophils, airway smooth muscle cells and other cancer cells measured using different microrheological methods [39,40]. $G^{\prime}$ exhibits a plateau at low frequencies until $20 \mathrm{~Hz}$ and then increases with frequency following a power law with exponent $a \simeq 0.8$. The viscous modulus $G^{\prime \prime}$ is smaller than the elastic modulus $G^{\prime}$ in the low frequency regime and increases with frequency until $G^{\prime \prime}$ dominates in the high-frequency domain.

An attempt to explain the power-law behavior of the microrheology spectrum of living cells has been made before [16]. By describing the cell as a soft glassy material [41], some rheological features can be assigned to the cytoskeletal organization and remodelling. A few authors $[11,16]$ measured the viscoelastic properties of cells using this model to explain their rheological data. A common parameter is the slope $(\sim 0.2)$ of $G^{\prime}$ and $G^{\prime \prime}$, at low frequencies, but a higher slope of 1 is found for $G^{\prime \prime}$ at higher frequencies, attributed to a viscous component. In our case, several regimes corresponding to different slopes coexist suggesting that the underlying processes that govern the rheology of cancer cells are not time-scale invariant. Thus it is not possible to describe the whole frequency domain with the SGR model. Explanations have been proposed in other works [36,37]. In these papers, it appears that the frequency dependent behavior can be explained by alternative mechanisms such as the collective behaviors of molecular non-covalent interactions of proteins.

Next, measurements were made at different cell locations, as shown in fig. 3. Data was obtained by indenting on top of the nucleus, perinucleus and periphery (the cell edge). Confocal microscopy images were made and positions of the indentations are shown, together with a height map of the actin microstructure.

Similar results are obtained for the three locations but the moduli increase as moving towards the edge ( $\mathrm{N}$ to $\mathrm{P}$ to E). We described our data with the simplified model and the fitting parameters of the moduli of T24 cells $\left(G_{N}^{0}, a\right.$ and $b$ only) are represented in fig. 4 . To link the parameters with the actin network microstructure, the mean fluorescence intensity of the actin (from confocal images) in the three regions was measured and represented also in fig. 4. One can observe that the intensity is higher at the periphery. This result is in agreement with other studies showing that the periphery of the cell is enriched in actin filaments [6].

If we compare the fluorescence intensity to the mechanical properties probed at the three different locations (fig. 3 and fig. 4), we find several interesting features. First the viscoelasticity of cancer cells is position-dependent and $G^{*}$ exhibits the same behavior at the three locations. However, the elastic plateau increases from the nucleus to the periphery, whereas the viscous modulus increases with frequency and becomes dominant at high frequencies. We

1 Rasband WS. 1997. ImageJ. Bethesda, MD: U.S. National Institutes of Health. 

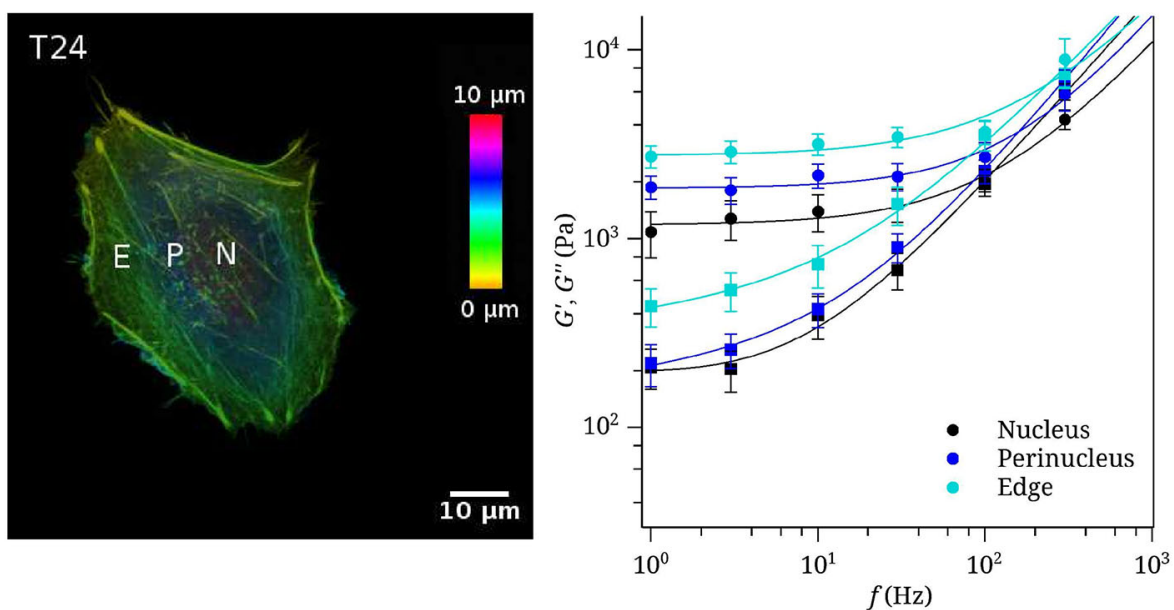

Fig. 3. Left: Confocal images of a single T24 cell. A z-projection with a color scale corresponding to the height (yellow is the basal side of the cell, and red is on top of the nucleus). Indentations are made at three locations: nucleus $(\mathrm{N})$, perinucleus $(\mathrm{P})$ and the edge (E). The cells are in a low migrating state. Right: Evolution of moduli $G^{\prime}$ (circle) and $G^{\prime \prime}$ (square) on the nucleus (black), perinucleus (blue) and edge of the cell (cyan); $N=20$ and error bars represent SEM. Curves were fitted with the model.
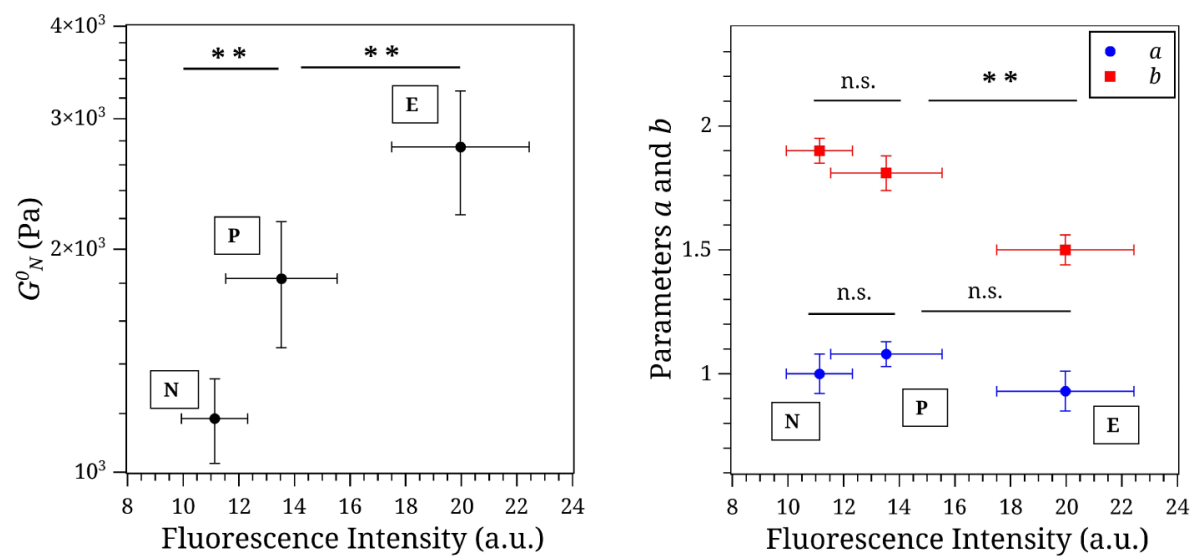

Fig. 4. Parameters $G_{N}^{0}, a$ and $b$ as functions of the fluorescence intensity (arbitrary units) of T24 cells at three different locations: nucleus $(\mathrm{N})$, perinucleus $(\mathrm{P})$ and the edge of the cell $(\mathrm{E}) ; N=20$ and error bars represent SEM. $p$-values were calculated using Student's unpaired t-test: ${ }^{* *} p<0.01$ represents significant difference between different locations and "n.s." means $p>0.05$ and there is no significant difference.

also observe that the gap between $G^{\prime}$ and $G^{\prime \prime}$ becomes more important at the periphery, suggesting the importance of elasticity due to the presence of actin fibres. Parameter $b\left(G^{\prime \prime} / G^{\prime}\right.$ at high frequencies $)$ is also a signature of an elasticity decrease as shown when plotted against fluorescence intensity. The parameter $G_{N}^{0}$ increases with fluorescence intensity confirming that the elasticity of the cell depends on the actin microstructure. The slope $a$ is relatively constant across the cell suggesting that the actin microstructure is not of major importance in the glassy transition. This slope could be explained by the collective behavior of non-covalent myosin bonds [36,37,42].

The spatial heterogeneity of the viscoelastic properties has been observed by Cai et al. [43]. They measured $G^{\prime}$ and $G^{\prime \prime}$ at two different locations on fibroblast cells (center and off-center) and found that the center of the cell is stiffer. Similarly, Rigato et al. [44] also noted that the centre of the cell is stiffer than the periphery. These findings can be explained by the fact that they investigated confined cell properties. Indeed cells were cultured on adhesive patterns, and were not free to form bundles of fibres at the periphery. This could explain the difference in behavior.

In our case, we find that the cell periphery is stiffer than the rest of the cell and the viscous component is lower. This behavior has also been found by Rebelo et al. [45] who investigated the mechanical properties at three locations on cancer and normal cells: peripheral region, intermediate height and cell body. Similarly to our results, they observe differences between responses of the cell body and the periphery of the cell: the cell edge has a higher Young modulus than the cell body. They explain this behavior by the structure and morphology of the periphery of each cell line (however they do not quantify the actin distribution as we did). 

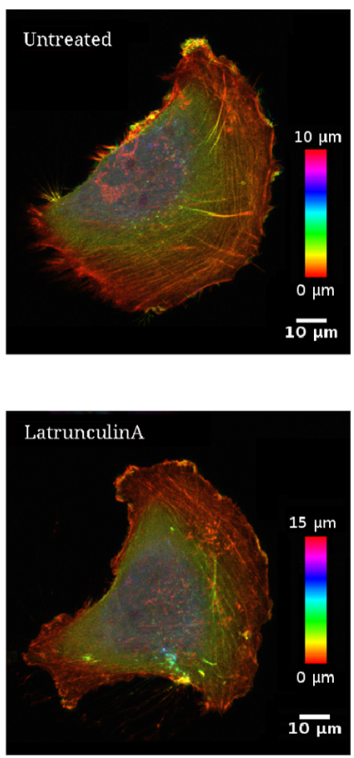
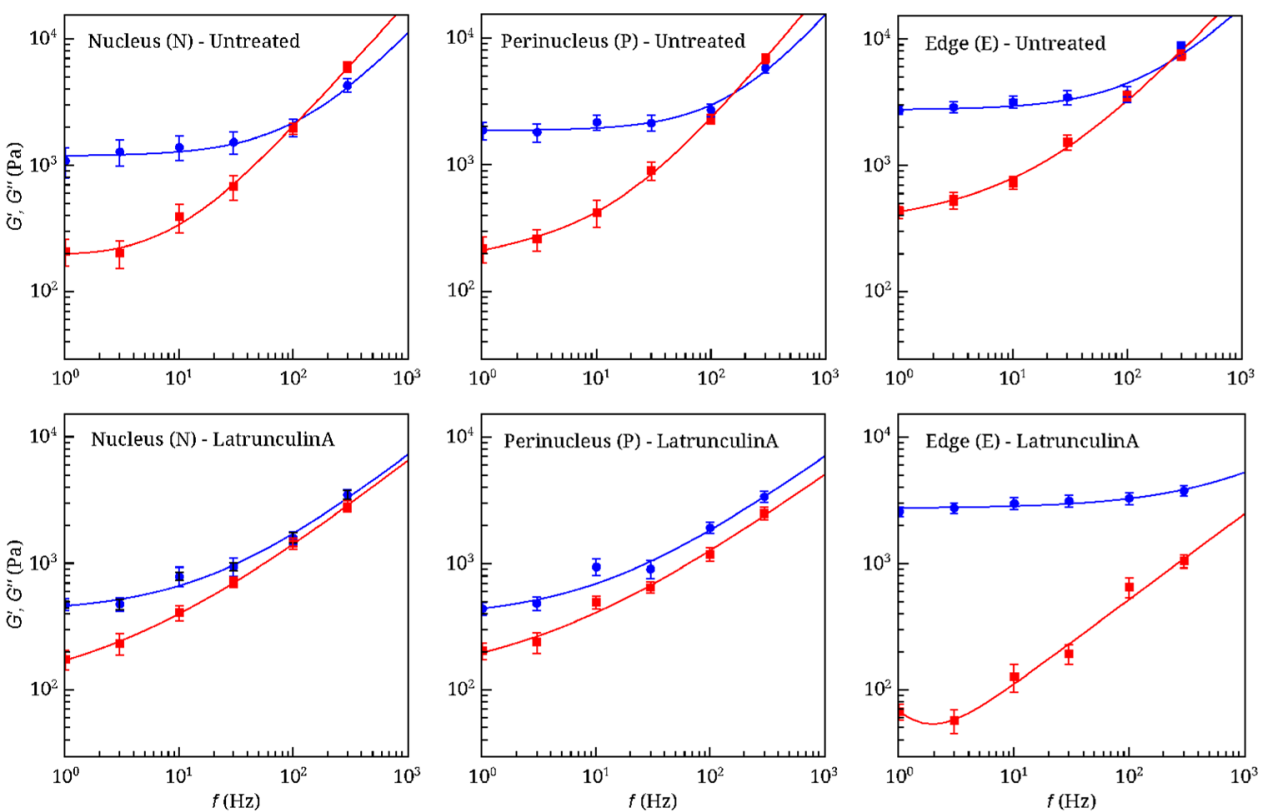

Fig. 5. Left: Confocal images of a single T24 cell before (left, above) and 20 minutes after Latrunculin A $0.1 \mu \mathrm{M}$ treatment (left, below). A $z$-projection was made and colors correspond to the height (red is the basal side of the cell, and pink is on top of the nucleus). Microrheological properties are measured at three locations: nucleus (N), perinucleus (P) and the edge (E). 20 confocal images have been made and usually cells are in a low migrating state. Right: Evolution of moduli $G^{\prime}$ (blue circle) and $G^{\prime \prime}$ (red square) on the nucleus, perinucleus and periphery of the cell for untreated (above) and treated conditions (below), $N=20$ and error bars represent SEM. Curves were fitted using the model (5)-(6). Parameters as well as the mean fluorescence intensity of each region are shown in fig. 6 and summarized in table 2 .

Disrupting the actin network will give us more information about how the cytoskeleton microstructure influences viscoelasticity and the model parameters.

\subsection{Influence of the actin network on cell mechanical properties}

The viscoelastic properties of cells measured by AFM are strongly associated with the actin network $[25,43]$ which is spatially heterogeneous and changes over time. To have a better understanding of the correlation between the actin microstructure and the mechanical properties, two major actin functions were inhibited: the actin polymerization using Latrunculin A and the acto-myosin contractility with Y27632.

\section{Inhibiting the actin polymerization with LatrunculinA}

Figure 5 shows the viscoelastic properties of T24 cells before and after treatment with Latrunculin $\mathrm{A}(0.1 \mu \mathrm{M})$ at three different locations. Measurements were carried out before $(t=0)$ and after treatment $(t=20 \mathrm{~min}$ and $t=30 \mathrm{~min})$. The reversibility of the treatment was verified (after the medium was rinsed, cells are stable for about 30 min and mechanical properties were recovered; data not shown). Confocal images corresponding to the two conditions (fig. 5) show that the actin filaments are indeed disrupted (for example, actin fibres that go from the bottom of the cell to the top disappear after treatment). However, there are still actin fibres at the basal side, this might be due to the low drug concentration we used (it was chosen so that the cell does not retract completely in order to perform AFM measurements).

The mean plateau modulus $G_{N}^{0}$ and corresponding fluorescence intensity are shown in fig. 6 . The parameters deduced from the model are summarized in table 2.

We first note that $G^{\prime}$ and $G^{\prime \prime}$ still increase with frequency after inhibition of the actin polymerization (fig. 5). These features are consistent with those observed in previous studies $[14,43,46]$. However, there are some notable differences. There is no crossing of $G^{\prime}$ and $G^{\prime \prime}$ at the high frequencies after inhibition of the actin polymerization, so the network remains elastic. The elastic plateau $G_{N}^{0}$ decreases at the centre of the cell and the perinucleus. However, at the periphery of the cell, $G_{N}^{0}$ remains constant even though the mean fluorescence intensity decreases (fig. 6). This may be due to the fact that the Latrunculin A concentration is small in our case. Finally, at the cell periphery, there is a huge decrease of $G^{\prime \prime}$ after treatment, in comparison with the other two locations. 

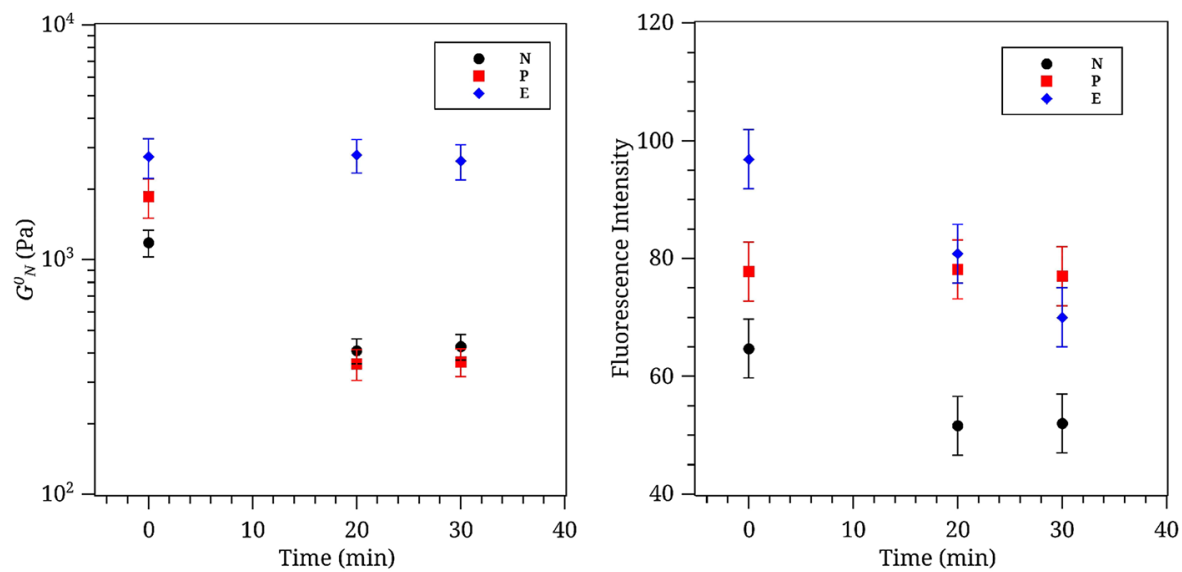

Fig. 6. $G_{N}^{0}$ (left) and fluorescence intensity (right) before $(t=0)$ and after treatment with Latrunculin $\mathrm{A} 0.1 \mu \mathrm{M}(t=20 \mathrm{~min}$, $t=30 \mathrm{~min}$ ). Parameters are summarized in table 2. $N=20$ and error bars represent SEM: When $G_{N}^{0}$ decreases (nucleus and perinucleus) and when the fluorescence intensity decreases (nucleus and edge), the differences are significant $(p<0.01)$.

Table 2. Fluorescence intensity (FI, arbitrary unit) and best-fitting values of parameters $G_{N}^{0}, n_{\mathrm{f}}, a$ and $b$ used in the model (5)(6) for T24 cells treated with Latrunculin A $(0.1 \mu \mathrm{M})$. Here only time $t=20$ min is shown; $N=20$ and all values are represented as mean \pm SEM.

\begin{tabular}{|c|c|c|c|}
\hline Cell location & Parameters & $t=0$ & $t=20 \mathrm{~min}$ \\
\hline \hline \multirow{4}{*}{ Nucleus (N) } & $\mathrm{FI}$ (a.u.) & $64 \pm 14$ & $51 \pm 11$ \\
\cline { 2 - 4 } & $G_{N}^{0}(\mathrm{~Pa})$ & $1182 \pm 154$ & $410 \pm 50$ \\
\cline { 2 - 4 } & $n_{\mathrm{f}}$ & $0.08 \pm 0.008$ & $-0.12 \pm 0.008$ \\
\cline { 2 - 4 } & $a$ & $1.00 \pm 0.03$ & $0.71 \pm 0.02$ \\
\cline { 2 - 4 } & $b$ & $1.90 \pm 0.08$ & $0.91 \pm 0.05$ \\
\hline \hline \multirow{3}{*}{ Perinucleus (P) } & $\mathrm{FI}($ a.u. $)$ & $77 \pm 19$ & $78 \pm 20$ \\
\cline { 2 - 4 } & $G_{N}^{0}(\mathrm{~Pa})$ & $1856 \pm 354$ & $360 \pm 54$ \\
\cline { 2 - 4 } & $n_{\mathrm{f}}$ & $-0.113 \pm 0.007$ & $-0.07 \pm 0.001$ \\
\cline { 2 - 4 } & $a$ & $1.08 \pm 0.02$ & $0.65 \pm 0.02$ \\
\cline { 2 - 4 } & $b$ & $1.80 \pm 0.08$ & $0.71 \pm 0.05$ \\
\hline \hline \multirow{3}{*}{ Edge (E) } & $\mathrm{FI}(\mathrm{a} . \mathrm{u})$. & $96 \pm 35$ & $80 \pm 35$ \\
\cline { 2 - 4 } & $G_{N}^{0}(\mathrm{~Pa})$ & $2749 \pm 524$ & $2796 \pm 454$ \\
\cline { 2 - 4 } & $n_{\mathrm{f}}$ & $-0.09 \pm 0.008$ & $0.29 \pm 0.008$ \\
\cline { 2 - 4 } & $a$ & $0.93 \pm 0.04$ & $0.67 \pm 0.04$ \\
\cline { 2 - 4 } & $b$ & $1.50 \pm 0.08$ & $0.97 \pm 0.05$ \\
\hline
\end{tabular}

All these observations suggest that the cell lamellipodium mechanics plays a specific role. The distribution of the actin cytoskeleton at the periphery is not sufficient to explain these features.

Inhibiting the actomyosin contractility with Y27632

Further investigations were carried out by inhibiting the acto-myosin contractility using Y27632. Only the model parameters are summarized in table 3.

When the acto-myosin contractility is inhibited, the cell cytoskeleton is not under tension anymore. The fluorescence intensity shows that the distribution of actin does not change. Nevertheless interesting comments can be made about the cell periphery properties, in particular $G_{N}^{0}$ considerably increases after treatment (table 3 ). The periphery becomes more rigid after inhibition. The values of $n_{\mathrm{f}}$ describe the $G^{\prime \prime}$ slope at low frequencies. In our case, these values are close to 0 or slightly $>0$ except for few cases in particular when using Y27632 at $t=20$ min at the cell edge. This result may be surprising, but it may be explained by the fact that Y27632 prevents the acto-myosin contractility, so 
Table 3. Fluorescence Intensity (FI, a.u.) and best-fitting values of parameters $G_{N}^{0}, n_{\mathrm{f}}, a$ and $b$ used in the model (5)-(6) for T24 cells treated with Y27632 $(20 \mu \mathrm{M}) ; N=10$ and all values are represented as mean \pm SEM.

\begin{tabular}{|c|c|c|c|}
\hline Cell location & Parameters & $t=0$ & $t=20 \min$ \\
\hline \multirow{5}{*}{ Nucleus (N) } & FI (a.u.) & $32 \pm 5$ & $36 \pm 5$ \\
\hline & $G_{N}^{0}(\mathrm{~Pa})$ & $1182 \pm 154$ & $480 \pm 31$ \\
\hline & $n_{\mathrm{f}}$ & $0.08 \pm 0.008$ & $-0.44 \pm 0.0068$ \\
\hline & $a$ & $1.00 \pm 0.03$ & $1.41 \pm 0.02$ \\
\hline & $b$ & $1.90 \pm 0.08$ & $1.1 \pm 0.01$ \\
\hline \multirow{5}{*}{ Perinucleus (P) } & FI (a.u.) & $46 \pm 4$ & $41 \pm 5$ \\
\hline & $G_{N}^{0}(\mathrm{~Pa})$ & $1856 \pm 354$ & $970 \pm 200$ \\
\hline & $n_{\mathrm{f}}$ & $-0.113 \pm 0.007$ & $-0.18 \pm 0.001$ \\
\hline & $a$ & $1.08 \pm 0.02$ & $0.83 \pm 0.05$ \\
\hline & $b$ & $1.80 \pm 0.08$ & $0.91 \pm 0.02$ \\
\hline \multirow{5}{*}{ Edge (E) } & FI (a.u.) & $54 \pm 8$ & $46 \pm 9$ \\
\hline & $G_{N}^{0}(\mathrm{~Pa})$ & $2749 \pm 524$ & $8563 \pm 500$ \\
\hline & $n_{\mathrm{f}}$ & $-0.09 \pm 0.008$ & $2.3 \pm 0.008$ \\
\hline & $a$ & $0.93 \pm 0.04$ & $0.89 \pm 0.04$ \\
\hline & $b$ & $1.50 \pm 0.08$ & $0.85 \pm 0.02$ \\
\hline
\end{tabular}

the nucleus and perinucleus regions get deprived from actin fibres which diffuse towards the lamellipodium, therefore elasticity increases. Recently, Labouesse et al. [47] suggested that there are myosin-independent forces, related to a passive elastic component, that contribute to the tension at the periphery. These findings can be related to our results since the inhibition of acto-myosin contractility increased the elasticity at the periphery. It could also be due to the presence of another relaxation mechanism associated to longer chains activated by proteins of the Rho kinase family that are not inhibited by Y27632, like Rac and cdc42 [48].

Direct influence of the actin network on the mechanical properties has been described in various studies [14,43, 49], however our results show that the cell periphery plays a unique role.

\subsection{Mechanical properties are a marker of cancer cell invasivity.}

To link invasiveness with the mechanical properties of cancer cells, we carried out dynamical experiments on three cell lines from human bladder carcinoma: RT112, T24 and J82 with increasing malignant potential, respectively. RT112 cells are less invasive than T24 and J82 [50]. Several studies have shown that the viscoelasticity of cancer cells can be a marker of their malignancy $[11,45]$. Since we found a particular behavior of the mechanical properties at the periphery (i.e. edge) of the cell, as also observed for some migrating cells [51], it is important to characterize the mechanical properties at the periphery of cancer cells of different invasiveness. Figure 7 shows the $G^{\prime}-G^{\prime \prime}$ curves of the three cell lines at three different positions.

In fig. 7, the elastic modulus $G^{\prime}$ decreases when malignancy increases. However, at the cell edge, $G_{N}^{0}(\mathrm{~T} 24)<$ $G_{N}^{0}(\mathrm{~J} 82)$ while on the nucleus it is the opposite, suggesting once again the unique properties of the cell periphery. To have a better understanding of the relationship between the actin microstructure of the three cell lines at different locations, the fluorescence intensity was linked with the parameters (table 4).

Parameter $G_{N}^{0}$ is plotted in fig. 8 for the three cell lines. An interesting behavior is found: for all cell lines, the elastic plateau is higher at the cell periphery, however the variations between each location are different. For example, the elastic plateau of J82 (the most invasive type) at the edge is 11 times higher than on the nucleus, whereas the ratio is 2.3 and 7 for T24 and RT112, respectively. Although the mechanical properties of bladder cancer cells seem to show interesting behaviors, they cannot be correlated with the fluorescence intensity, i.e. the actin microstructure. This can be due to the fact that the shape of each cell line is inherently different. Indeed, J82 cells are known to show an elongated morphology [52]. 

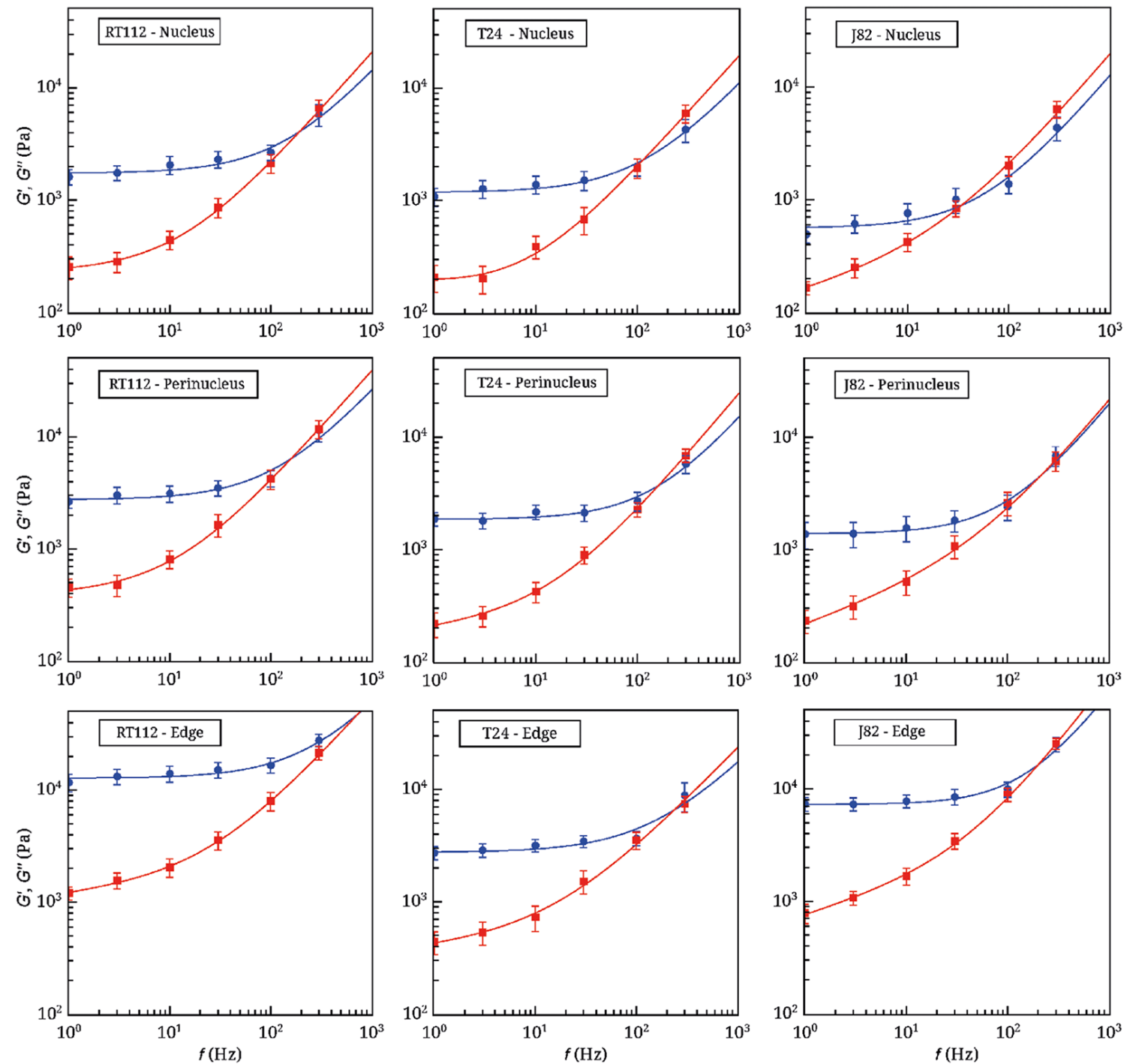

Fig. 7. Evolution of $G^{\prime}$ (blue circle) and $G^{\prime \prime}$ (red square) on the nucleus (N), perinucleus (P) and edge (E) - top to bottom row - of three cancer cell lines: RT112, T24 and J82 (left to right, respectively, $N=10, N=20, N=10$, error bars represent SEM). Curves were fitted with the model (5)-(6); $G_{N}^{0}$ is plotted in fig. 8 and the other parameters are summarized in table 4.

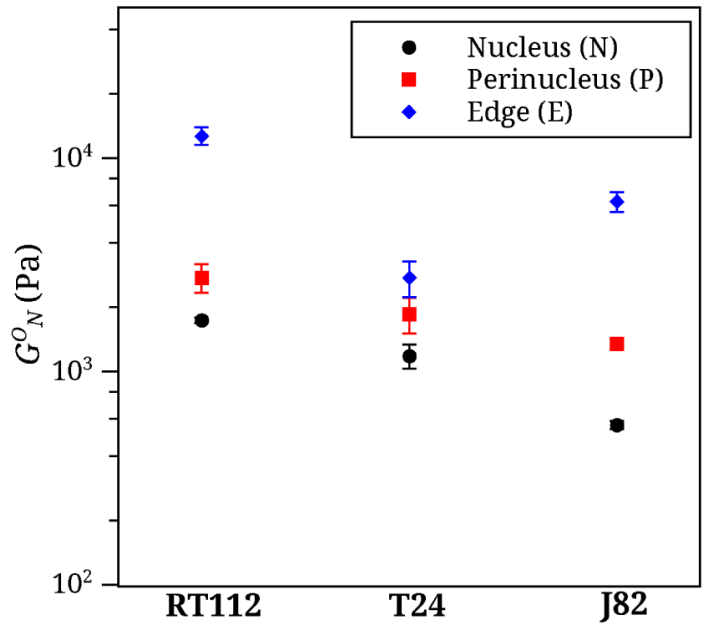

Fig. 8. Parameter $G_{N}^{0}$ (three cell lines: RT112, T24, J82) on nucleus (N), perinucleus (P) and cell edge (E). The other parameters are summarized in table 4 . The differences between $G_{N}^{0}$ of all different cell lines at all locations are significant $(p<0.01)$. 
Table 4. Fluorescence intensity (FI, a.u.) and the best-fitting values of parameters $G_{N}^{0}, n_{\mathrm{f}}, a$ and $b$ used in the model (5)-(6) for cancer cells of different invasivity RT112, T24 and J82 (respectively, $N=10, N=20, N=10$, all values are represented as mean $\pm \mathrm{SEM})$. The transition frequency $f_{\mathrm{T}}$ is determined experimentally.

\begin{tabular}{|c|c|c|c|c|}
\hline Parameters & Cell line & Nucleus (N) & Perinucleus (P) & Cell edge (E) \\
\hline \multirow{3}{*}{ FI (a.u.) } & RT112 & $26 \pm 5$ & $20 \pm 5$ & $35 \pm 5$ \\
\hline & $\mathrm{T} 24$ & $11 \pm 1.1$ & $13.5 \pm 2.0$ & $20 \pm 2.4$ \\
\hline & J82 & $49.8 \pm 11$ & $61 \pm 15$ & $80 \pm 20$ \\
\hline \multirow{3}{*}{$G_{N}^{0}(\mathrm{~Pa})$} & RT112 & $1738 \pm 54$ & $2756 \pm 102$ & $12701 \pm 1200$ \\
\hline & $\mathrm{T} 24$ & $1182 \pm 154$ & $1856 \pm 120$ & $2749 \pm 524$ \\
\hline & J82 & $561 \pm 24$ & $1350 \pm 45$ & $6254 \pm 652$ \\
\hline \multirow{3}{*}{$n_{\mathrm{f}}$} & RT112 & $-0.01 \pm 0.006$ & $-0.27 \pm 0.005$ & $-0.1 \pm 0.005$ \\
\hline & $\mathrm{T} 24$ & $0.08 \pm 0.005$ & $-0.113 \pm 0.005$ & $-0.09 \pm 0.005$ \\
\hline & J82 & $-0.255 \pm 0.005$ & $-0.3 \pm 0.005$ & $-0.26 \pm 0.005$ \\
\hline \multirow{3}{*}{$a$} & RT112 & $1.01 \pm 0.05$ & $1.01 \pm 0.02$ & $1.01 \pm 0.02$ \\
\hline & $\mathrm{T} 24$ & $1.00 \pm 0.01$ & $1.08 \pm 0.02$ & $0.93 \pm 0.04$ \\
\hline & J82 & $1.06 \pm 0.01$ & $1.13 \pm 0.05$ & $1.12 \pm 0.06$ \\
\hline \multirow{3}{*}{$b$} & RT112 & $1.63 \pm 0.05$ & $1.59 \pm 0.05$ & $1.3 \pm 0.02$ \\
\hline & $\mathrm{T} 24$ & $1.9 \pm 0.06$ & $1.8 \pm 0.02$ & $1.5 \pm 0.04$ \\
\hline & J82 & $1.55 \pm 0.05$ & $1.06 \pm 0.05$ & $1.51 \pm 0.06$ \\
\hline \multirow{3}{*}{$f_{\mathrm{T}}(\mathrm{Hz})$} & RT112 & $226 \pm 10$ & $160 \pm 15$ & $879 \pm 21$ \\
\hline & $\mathrm{T} 24$ & $104 \pm 5$ & $170 \pm 15$ & $220 \pm 5$ \\
\hline & $\mathrm{J} 82$ & $38 \pm 5$ & $205 \pm 20$ & $194 \pm 5$ \\
\hline
\end{tabular}

Another important parameter to deduce from the AFM measurements is the transition frequency $f_{\mathrm{T}}$ that corresponds to the crossing of the $G^{\prime}$ and $G^{\prime \prime}$ moduli (table 4, fig. 8). On the nucleus, $f_{\mathrm{T}}$ shifts to the lower frequencies as the invasivity increases (fig. 7). This is well correlated to the decrease of the plateau modulus $G_{N}^{0}$. The transition frequency $f_{\mathrm{T}}$ seems to be a signature of the invasiveness on the nucleus. On the perinucleus, there is no significant difference. As for the periphery, we find that $f_{\mathrm{T}}$ decreases when invasivity increases (T24 and J82 have close malignancy potential). On the nucleus and the edge, cells with higher invasivity have a lower transition frequency. This suggests once again that the properties at the periphery are critical, and can be used as a marker of malignancy.

Several researchers have investigated the relationship between invasivity and cellular mechanics. Ramos et al. [20] investigated the elastic properties of non-malignant and cancerous bladder cell lines using the AFM. They found that normal cells are stiffer than cancer cells. The viscoelastic properties of different cell lines (malignant and benign) were also investigated by Rother et al. [11] by AFM who showed that the loss tangent $\left(G^{\prime \prime} / G^{\prime}\right)$ at $100 \mathrm{~Hz}$ increases with the metastatic potential of cancer cells and can be used as a marker of invasivity. We have also calculated the loss tangent at $100 \mathrm{~Hz}$ of the three cell lines on the nucleus (data not shown) and we find similar findings. Further local measurements reported the cell edge viscoelastic properties. We found that the elastic plateau $G_{N}^{0}$ (obtained at low frequencies) is a marker of metastatic potential, since this parameter decreases when invasivity increases. However, on the periphery of invasive cells, $G_{N}^{0}(\mathrm{~J} 82)>G_{N}^{0}(\mathrm{~T} 24)$ which suggests that J82 has particular cell edge properties. In addition, a transition frequency $f_{\mathrm{T}}$ was introduced here in relation with our model. This frequency decreases with malignancy on the nucleus and the edge and can therefore be used as a pertinent marker of cell invasiveness.

\section{Conclusions}

A new method for characterizing the rheology of cancer cells has been developped in the range [1-300 Hz], together with the use of a realistic model containing a few parameters than can be used for describing changes in cell rheology or invasivity. By comparing the actin microstructure to the viscoelasticity of cancer cells by AFM, we showed that local properties are important and related to the actin organization in bladder cancer cell lines. In particular, we emphasized the higher rigidity of the cell periphery. 
To reach a better understanding of the relationship between the local viscoelastic properties and the actin microstructure, we disrupted the actin polymerization and the actomyosin contractility using two drugs. Latrunculin A was found to decrease the $\left(G^{\prime}, G^{\prime \prime}\right)$ moduli on the nucleus and perinucleus but not at the periphery. Y27632 had a similar effect but increased the plateau modulus $G_{N}^{0}$. This seems to reveal the presence of a passive elastic tension at the periphery, as mentioned previously [47] or it can be due to a reorganisation of actin binding proteins from the Rho family not inhibited by the drug [48].

Similarly, three cell lines of different invasivity were tested using this method and it was found that the elastic plateau $G_{N}^{0}$ and the transition frequency $f_{\mathrm{T}}$ both decreased for higher invasive cells. However, at the periphery of the most invasive cancer cells (J82), a higher elastic plateau was observed. This new result on the cell periphery may be of importance when cells are transmigrating through the vascular wall during metastasis, since it can enable them to exert higher tractions to pass through the endothelium lining.

We thank the ANR for grant No. 12-BS09-020-01 (TRANSMIG), the Nanoscience foundation for support of the AFM platform. This work has been partially supported by the LabeX Tec21 (Investissements d'Avenir - grant agreement No. ANR-11-LABX0030).

Open Access This is an open access article distributed under the terms of the Creative Commons Attribution License (http://creativecommons.org/licenses/by/4.0), which permits unrestricted use, distribution, and reproduction in any medium, provided the original work is properly cited.

\section{References}

1. H. Yamaguchi, J. Condeelis, Biochim. Biophys. Acta 1773, 642 (2007).

2. G. Weder, M. HendriksBalk, R. Smajda, D. Rimoldi, M. Liley, H. Heinzelmann, A. Meister, A. Mariotti, Nanomedicine 10, $141(2014)$.

3. G. Bao, S. Suresh, Nat. Mater. 2, 715 (2003).

4. C. Zhu, G. Bao, N. Wang, Annu. Rev. Biomed. Eng. 2, 189 (2000).

5. V.M. Laurent, R. Fodil, P. Canadas, S. Féréol, B. Louis, E. Planus, D. Isabey, Ann. Biomed. Eng. 31, 1263 (2003).

6. S. Suresh, Acta Biomater. 3, 413 (2007).

7. E. Moeendarbary, A.R. Harris, Wiley Interdiscip. Rev.: Syst. Biol. Med. 6, 371 (2014).

8. G. Zhang, M. Long, Z. Wu, W. Yu, World J. Gastroenterol. 8, 243 (2002).

9. M. Lekka, P. Laidler, D. Gil, J. Lekki, Z. Stachura, A.Z. Hrynkiewicz, Eur. Biophys. J. 28, 312 (1999).

10. S.E. Cross, Y. Jin, J. Tondre, R. Wong, J. Rao, J. Gimzewski, Nanotechnology 19, 384003 (2008).

11. J. Rother, H. Noding, I. Mey, A. Janshoff, Open Biol. 4, 140046 (2014).

12. B. Fabry, G.N. Maksym, J.P. Butler, M. Glogauer, D. Navajas, N.A. Taback, E.J. Millet, J.J. Fredberg, Phys. Rev. E 68, $041914(2003)$.

13. S. Yamada, D. Wirtz, S.C. Kuo, Biophys. J. 78, 1736 (2000).

14. K.M. Van Citters, B.D. Hoffman, G. Massiera, J.C. Crocker, Biophys. J. 91, 3946 (2006).

15. R.E. Mahaffy, C.K. Shih, F.C. MacKintosh, J. Käs, Phys. Rev. Lett. 85, 880 (2000).

16. J. Alcaraz, L. Buscemi, M. Grabulosa, X. Trepat, B. Fabry, R. Farre, D. Navajas, Biophys J. 84, 2071 (2003).

17. J. Guck, R. Ananthakrishnan, H. Mahmood, T.J. Moon, C.C. Cunningham, J. Käs, Biophys. J. 81, 767 (2001).

18. R.E. Mahaffy, S. Park, E. Gerde, J. Käs, C.K. Shih, Biophys. J. 86, 880 (2004).

19. F. Rico, P. Roca-Cusachs, N. Gavara, R. Farre, M. Rotger, D. Navajas, Phys. Rev. E 72, 021914 (2005).

20. J.R. Ramos, J. Pabijan, R. Garcia, M. Lekka, Beilstein J. Nanotechnol. 5, 447 (2014).

21. G.H. Koenderink, Z. Dogic, F. Nakamura, P.M. Bendix, F.C. MacKintosh, J.H. Hartwig, T.P. Stossel, D.A. Weitz, Proc. Natl. Acad. Sci. U.S.A. 106, $15192(2009)$

22. R. Levayer, T. Lecuit, Trends Cell Biol. 22, 61 (2012).

23. J. Prost, F. Jülicher, J.-F. Joanny, Nature Phys. 11, 111 (2015).

24. M. Guo, A.J. Ehrlicher, M.H. Jensen, M. Renz, J.R. Moore, R.D. Goldman, J. Lippincott-Schwartz, F.C. Mackintosh, D.A. Weitz, Cell 158, 822 (2014).

25. C. Rotsch, M. Radmacher, Biophys. J. 78, 520 (2000).

26. T. Wakatsuki, B. Schwab, N.C. Thompson, E.L. Elson, J. Cell Sci. 114, 1025 (2001).

27. J.C. Martens, M. Radmacher, Pflugers Arch. 456, 95 (2008).

28. M.F. Olson, E. Sahai, Clin. Exp. Metastasis 26, 273 (2009).

29. E.G. Yarmola, T. Somasundaram, T.A. Boring, I. Spector, M.R. Bubb, J. Biol. Chem. 275, 28120 (2000).

30. C.B. O'Connell, M.J. Tyska, M.S. Mooseker, Biochim. Biophys. Acta 1773, 615 (2007).

31. S. Narumiya, T. Ishizaki, M. Uehata, Methods Enzymol. 325, 273 (2000).

32. G.G. Bilodeau, J.Appl. Mech. 59, 519 (1992).

33. Y. Abidine, V.M. Laurent, R. Michel, A. Duperray, L.I. Palade, C. Verdier, EPL 109, 38003 (2015).

34. E. Dimitriadis, F. Horkay, J. Maresca, B. Kachar, R.S. Chadwick, Biophys. J. 82, 2798 (2002). 
35. J.A. Santos, L.M. Rebelo, A.C. Araujo, E.B. Barros, J.S. de Sousa, Soft Matter 8, 4441 (2012).

36. D. Stamenovic, N. Rosenblatt, M. Montoya-Zavala, B.D. Matthews, S. Hu, B. Suki, N. Wang, D.E. Ingber, Biophys. J. 93, L39 (2007).

37. F. Chowdhury, S. Na, O. Collin, B. Tay, F. Li, T. Tanaka, D.E. Leckband, N. Wang, Biophys. J. 95, 5719 (2008).

38. S.L. Gupton, C.M. Waterman-Storer, Cell 125, 1361 (2006).

39. B.A. Smith, B. Tolloczko, J.G. Martin, P. Grutter, Biophys. J. 88, 2994 (2005).

40. B. Fabry, G.N. Maksym, J.P. Butler, M. Glogauer, D. Navajas, J.J. Fredberg, Phys. Rev. Lett. 87, 148102 (2001).

41. P. Sollich, Phys. Rev. E 58, 738 (1998).

42. J. Etienne, J. Fouchard, D. Mitrossilis, N. Bufi, P. Durand-Smet, A. Asnacios, Proc. Natl. Acad. Sci. U.S.A. 112, 2740 (2015).

43. P. Cai, Y. Mizutani, M. Tsuchiya, J.M. Maloney, B. Fabry, K.J. Van Vliet, T. Okajima, Biophys. J. 105, 1093 (2013).

44. A. Rigato, F. Rico, F. Eghiaian, M. Piel, S. Scheuring, ACS Nano 9, 5846 (2015).

45. L.M. Rebelo, J.S. de Sousa, J.M. Filho, M. Radmacher, Nanotechnology 24, 055105 (2013).

46. S. Hiratsuka, Y. Mizutani, M. Tsuchiya, K. Kawahara, H. Tokumoto, T. Okajima, Ultramicroscopy 109, 937 (2009).

47. C. Labouesse, A.B Verkhovsky, J.J. Meister, C. Gabella, B. Vianay, Biophys. J. 108, 2437 (2015).

48. M. Raftopoulou, A. Hall, Dev. Biol. 265, 23 (2004).

49. L. Lu, S.J. Oswald, H. Ngu, F.C-P. Yin, Biophys. J. 95, 6060 (2008).

50. P. Champelovier, A. Simon, C. Garrel, G. Levacher, V. Praloran, D. Seigneurin, Clin. Cancer Res. 9, 4562 (2003).

51. V.M. Laurent, S. Kasas, A. Yersin, T.E. Schäffer, S. Catsicas, G. Dietler, A.B. Verkhovsky, J.-J. Meister, Biophys. J. 89, $667(2005)$.

52. V. Peschetola, V.M. Laurent, A. Duperray, R. Michel, D. Ambrosi, L. Preziosi, C. Verdier, Cytoskeleton 70, 201 (2013). 